\title{
Hydrothermal Synthesis and Responsive Characteristics of Hierarchical Zinc Oxide Nanoflowers to Sulfur Dioxide
}

\author{
Qu Zhou, ${ }^{1}$ Bo Xie, ${ }^{2}$ Lingfeng Jin, ${ }^{2}$ Weigen Chen, ${ }^{2}$ and Jian $\mathrm{Li}^{2}$ \\ ${ }^{1}$ College of Engineering and Technology, Southwest University, Chongqing 400715, China \\ ${ }^{2}$ State Key Laboratory of Power Transmission Equipment \& System Security and New Technology, Chongqing University, \\ Chongqing 400030, China \\ Correspondence should be addressed to Qu Zhou; zhouqu@swu.edu.cn
}

Received 4 December 2015; Revised 19 February 2016; Accepted 21 February 2016

Academic Editor: Thomas Thundat

Copyright (C) 2016 Qu Zhou et al. This is an open access article distributed under the Creative Commons Attribution License, which permits unrestricted use, distribution, and reproduction in any medium, provided the original work is properly cited.

\begin{abstract}
Sulfur dioxide, $\mathrm{SO}_{2}$, is one of the most important decomposition byproducts of sulfur hexafluoride, $\mathrm{SF}_{6}$, under partial discharge in GIS apparatus. The sensing performances of semiconductor gas sensors can be improved by morphology tailoring. This paper reported the synthesis method, structural characterization, and $\mathrm{SO}_{2}$ responsive characteristics of hierarchical flower-shaped $\mathrm{ZnO}$ nanostructures. Hierarchical $\mathrm{ZnO}$ nanoflowers were successfully prepared via a facile and simple hydrothermal method and characterized by X-ray powder diffraction, scanning electron microscopy, energy dispersive X-ray spectroscopy, and X-ray photoelectron spectroscopy, respectively. Planar chemical gas sensor was fabricated and its responsive characteristics towards $\mathrm{SO}_{2}$ were systematically performed. The optimum operating temperature of the fabricated sensor was measured to be about $260^{\circ} \mathrm{C}$, and the corresponding maximum responses were 16.72 and 26.14 to 30 and $60 \mathrm{ppm}$ of $\mathrm{SO}_{2}$. Its saturated gas concentration was $2000 \mathrm{ppm}$ with a response value of 67.41. Moreover, a quick response and recovery feature ( $7 \mathrm{~s}$ and $8 \mathrm{~s}$ versus $80 \mathrm{ppm}$ of $\mathrm{SO}_{2}$ ) and good stability were also observed. All results indicate that the proposed sensor is a promising candidate for detecting $\mathrm{SF}_{6}$ decomposition byproduct $\mathrm{SO}_{2}$.
\end{abstract}

\section{Introduction}

Sulfur hexafluoride, $\mathrm{SF}_{6}$, is a typical kind of colorless, tasteless, nontoxic, and nonflammable inert gas under general conditions with an extreme high thermal decomposition temperature $[1,2]$. With the prominent advantages of small floor space, high reliability and stability, excellent insulation strength, non-smeary oil, and lower maintenance cost, gas insulated switchgear (GIS) apparatus filled with pressurized $\mathrm{SF}_{6}$ gas are widely used in electrical power system in recent decades. Inevitable defects existed during the design, manufacture, transportation, installation, and operation processes of a GIS system [3]. These internal insulation defects, like metal burrs or suspended particles, may cause insulation degradation and even partial discharge in the long-term service cycle. If partial discharge occurs in GIS, $\mathrm{SF}_{6}$ gas molecules firstly decompose into some low-fluorine sulfides and then react with trace oxygen and water vapor to generate various kinds of decomposition byproducts, for instance,
$\mathrm{SO}_{2}, \mathrm{SOF}_{2}, \mathrm{SO}_{2} \mathrm{~F}_{2}, \mathrm{SOF}_{4}, \mathrm{H}_{2} \mathrm{~S}$, and $\mathrm{HF}[4,5]$. These decomposition byproducts would speed up the aging of insulation material and the corrosion of metal, eventually resulting in faults that happened in GIS.

Recent researches at home and abroad demonstrated that the composition and concentration of $\mathrm{SF}_{6}$ decomposition byproducts are closely related to the insulation status of GIS. Monitoring and analyzing the component contents of these characteristic decomposition products and their generation rates are one of the most effective and convenient methods for GIS condition assessment and fault diagnosis [3,5]. Till now, the main sensing approaches employed for recognizing $\mathrm{SF}_{6}$ decomposition byproducts are gas chromatography $[1,2]$, infrared absorption spectrometry [6,7], and metal oxide semiconductor $[8,9]$. Gas chromatography is mainly used for offline testing in laboratory and cross sensitivity between $\mathrm{SF}_{6}$ and its decomposition byproducts exists for infrared absorption spectrometry [10, 11]. With the advantages of simple fabrication, low cost, high response, rapid response 
and recovery time, and facile integration, metal oxide semiconductor like n-type $\mathrm{ZnO}$ [12-14] or n-type $\mathrm{SnO}_{2}$ [15-18] may represent the most promising sensing technology for sensing $\mathrm{SF}_{6}$ decomposition byproducts. However, due to the deficiencies of low gas response and high working temperature, the application of using metal oxide semiconductor based sensors detecting $\mathrm{SF}_{6}$ decomposition byproducts is greatly limited $[8,10]$.

Hence, in this study, we prepare hierarchical $\mathrm{ZnO}$ nanoflowers through a simple hydrothermal process and report systematically their responsive characteristics to $\mathrm{SO}_{2}$, one of the most important decomposition byproducts of $\mathrm{SF}_{6}$ under partial discharge in GIS apparatus.

\section{Experimental Details}

All chemicals were analytical grade reagents purchased from Chongqing Chuandong Chemical Reagent Co. Ltd. and used as received without any further purification. Hierarchical flower-shaped $\mathrm{ZnO}$ nanostructures were synthesized with a simple, facile, and environment-friendly hydrothermal method. The detailed synthesis processes were represented as follows.

Typically, $2.0 \mathrm{mmol}$ zinc nitrate hexahydrate, $\mathrm{Zn}\left(\mathrm{NO}_{3}\right)_{2}$. $6 \mathrm{H}_{2} \mathrm{O}, 4.0 \mathrm{mmol} \mathrm{NH}_{4} \mathrm{OH}, 28 \mathrm{wt} \% \mathrm{NH}_{3}$ in $\mathrm{H}_{2} \mathrm{O}, 0.83 \mathrm{~g} \mathrm{CTAB}$, $30 \mathrm{~mL}$ absolute ethanol, and $30 \mathrm{~mL}$ distilled water were mixed together with intense magnetic stirring, which were subsequently transferred into a Teflon autoclave, sealed, and heated at $160^{\circ} \mathrm{C}$ for $24 \mathrm{~h}$ in an electric furnace. After reaction, the autoclave was cooled to room temperature naturally, harvested by centrifugation, washed with distilled water and absolute ethanol several times to remove the ions possibly remaining in the final product, and finally dried at $80^{\circ} \mathrm{C}$ in air for further use.

The crystalline structures of the as-prepared $\mathrm{ZnO}$ samples were performed by X-ray powder diffraction (XRD, Rigaku D/Max-1200X, Japan) with $\mathrm{Cu} \mathrm{K \alpha}$ radiation operated at $40 \mathrm{kV}$ and $200 \mathrm{~mA}$ and a scanning rate of $0.02^{\circ} \mathrm{s}^{-1}$ from $20^{\circ}$ to $80^{\circ}$ [12]. Surface morphologies and microstructures of the as-prepared nanostructures were observed with a Nova 400 Nano field emission scanning electron microscope (FESEM, FEI, Hillsboro, OR, USA) equipped with an energy dispersive X-ray spectroscopy (EDS) [15]. X-ray photoelectron spectroscopy (XPS) was performed on an ESCLAB MKII using monochromatic $\mathrm{Al} \mathrm{K} \alpha$ as the $\mathrm{X}$-ray exciting source to investigate the chemical state of elements existing in the samples.

Planar chemical gas sensors based on the as-prepared powders were fabricated with screen-printing technique, and the planar ceramic substrates were purchased from Beijing Elite Tech Co., Ltd., China [12]. Firstly, the asprepared nanostructures were mixed with deionized water and absolute ethanol in a weight ratio of $10: 2: 1$ to form a suspension. Then it was subsequently screen-printed onto the planar ceramic substrate to form a sensing film with a thickness of about $50 \mu \mathrm{m}$. Finally, the fabricated sensor was dried in air at $100^{\circ} \mathrm{C}$ to volatilize the organic solvent and further aged in an aging test chamber for $36 \mathrm{~h}$. Responsive characteristics of the fabricated sensors to $\mathrm{SO}_{2}$ were measured

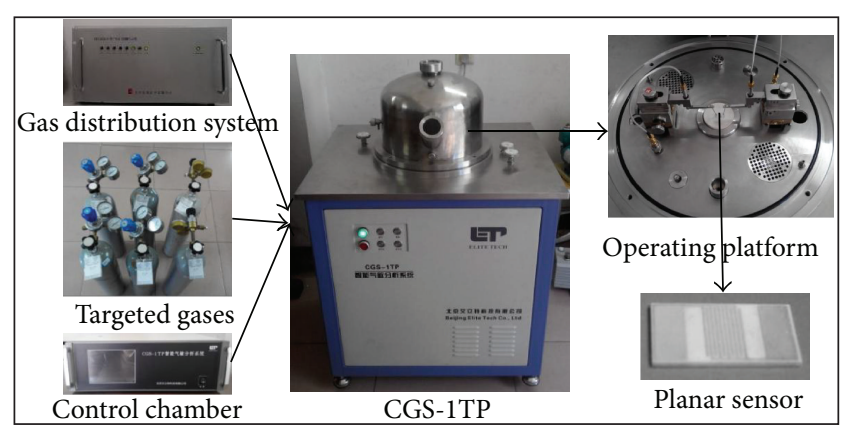

FIGURE 1: Schematic diagram of the CGS-1TP gas sensing analysis system.

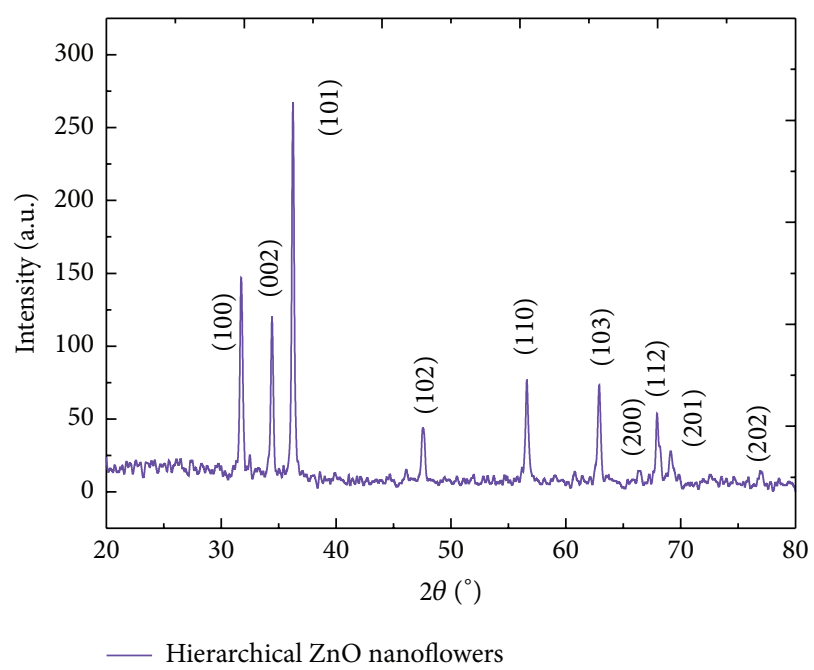

FIGURE 2: Typical XRD patterns of the synthesized hierarchical $\mathrm{ZnO}$ nanoflowers.

and automatically recorded by a CGS-1TP intelligent gas sensing analysis system, purchased from Beijing Elite Tech Co., Ltd., China. Figure 1 shows the schematic diagram of the CGS-1TP gas sensing analysis system [15].

In this study, gas response of the sensor to $\mathrm{SF}_{6}$ partial discharge decomposition byproduct $\mathrm{SO}_{2}$ was defined as $S=$ $R_{a} / R_{g}[19,20]$, where $R_{a}$ denotes the resistance value of the sensor in pure $\mathrm{N}_{2}$ and $R_{g}$ in certain concentration of target gas mixed with pure $\mathrm{N}_{2}$. The response and recovery time were defined as the time required by the sensor to achieve $90 \%$ of the total resistance change in the case of gas adsorption or gas desorption [21, 22]. All measurements were repeated several times to ensure the repeatability of the sensor.

\section{Results and Discussion}

The crystalline phases and structures of the as-prepared hierarchical $\mathrm{ZnO}$ nanostructures after annealing at $600^{\circ} \mathrm{C}$ for $5 \mathrm{~h}$ were characterized by the X-ray powder diffraction measurement and shown in Figure 2. One can clearly see from this figure that all the peaks are corresponding to wurtzite hexagonal $\mathrm{ZnO}$ structure given in the standard data file of JCPDS. 36-1451 [23]. The prominent peaks at $31.7^{\circ}$, 


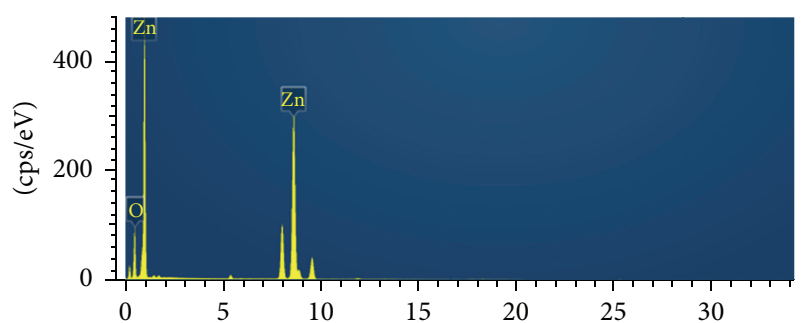

FIGURE 3: EDS spectrum of the synthesized hierarchical $\mathrm{ZnO}$ nanoflowers.

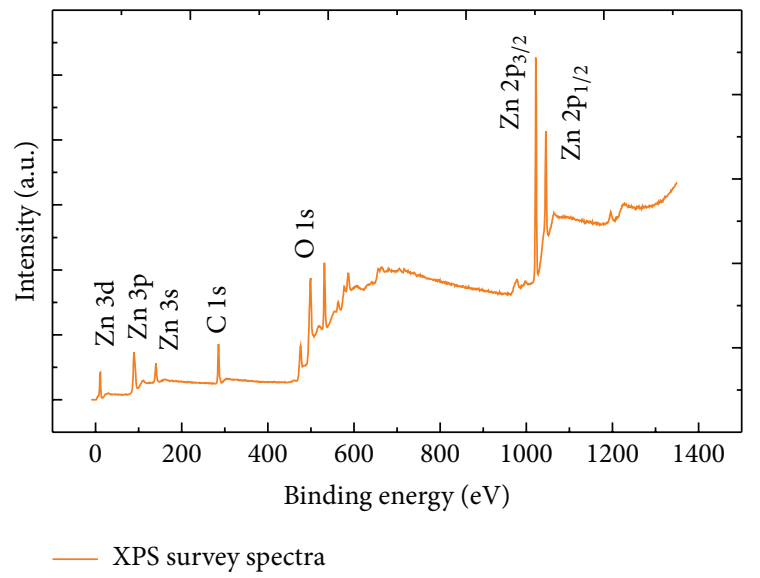

FIGURE 4: XPS survey spectra of the synthesized hierarchical $\mathrm{ZnO}$ nanoflowers.

$34.4^{\circ}$, and $36.2^{\circ}$ can be well indexed as the (100), (002), and (101) planes of wurtzite hexagonal $\mathrm{ZnO}$, respectively [24, 25]. No other diffraction peaks from any impurities are detected, indicating a high purity of the as-prepared samples.

Energy dispersive X-ray spectroscopy measurement was further performed to investigate the element components of the as-synthesized samples. Figure 3 demonstrates the EDS spectrum of the synthesized $\mathrm{ZnO}$ nanostructures. As shown in Figure 3, only $\mathrm{Zn}$ and $\mathrm{O}$ element peaks were observed, which confirms the availability of $\mathrm{Zn}$ and $\mathrm{O}$ in the synthesized samples [25-27].

To further investigate the composition and the chemical state of the elements existing in the synthesized $\mathrm{ZnO}$ nanostructures, X-ray photoelectron spectroscopy XPS measurement was performed and collected. Adventitious hydrocarbon $\mathrm{C} 1 \mathrm{~s}$ binding energy at $285 \mathrm{eV}$ was used as a reference to correct the energy shift of O 1s. The XPS wide survey spectrum of the synthesized hierarchical samples as shown in Figure 4 confirms the existence of $\mathrm{Zn}, \mathrm{O}$, and $\mathrm{C}$. The binding energies at $1022.3 \mathrm{eV}$ and $1044.9 \mathrm{eV}$ could be well identified as $\mathrm{Zn} 2 \mathrm{p}_{3 / 2}$ and $\mathrm{Zn} 2 \mathrm{p}_{1 / 2}$, which could be attributed to $\mathrm{Zn}^{2+}$ ions [25-27]. Thus, based on these XRD, EDS, and XPS results, we can draw a conclusion that pure $\mathrm{ZnO}$ nanostructures have been successfully synthesized with the current route [23].

The surface structures and morphologies of the assynthesized $\mathrm{ZnO}$ nanostructures were performed by FESEM. As illustrated in Figure 5 the panoramic image of the asprepared hierarchical $\mathrm{ZnO}$ nanostructures is in flower shape,

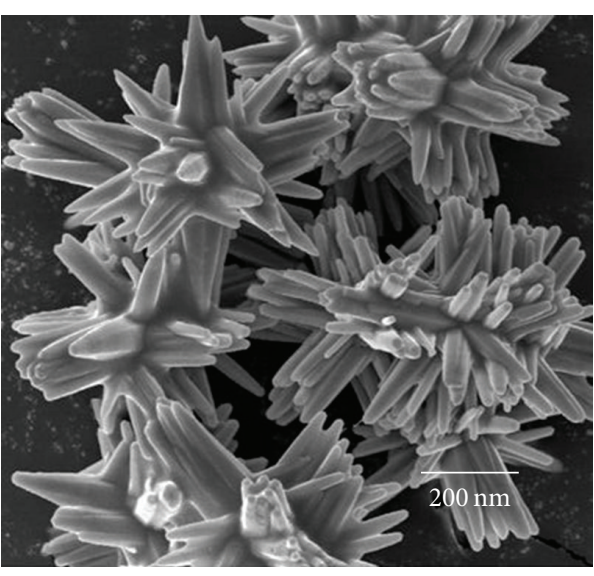

FIGURE 5: FESEM image of the synthesized hierarchical $\mathrm{ZnO}$ nanoflowers.

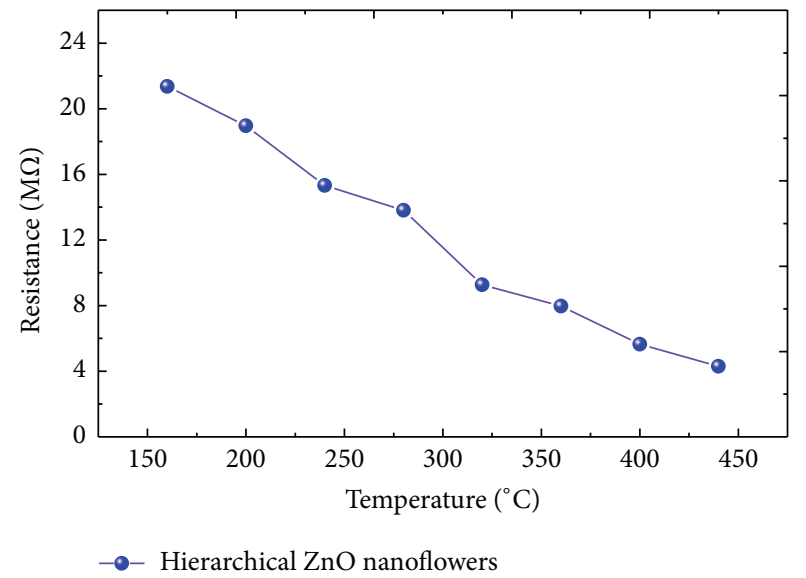

FIGURE 6: Resistance of the hierarchical $\mathrm{ZnO}$ nanoflowers sensor versus operating temperature.

which consisted of a large number of nanorods. These nanorods are rectangular with high uniformity in shape and size. No other morphologies have been detected, suggesting a high yield of the flower-like $\mathrm{ZnO}$ nanostructures.

Figure 6 shows the electrical resistance curve of the sensor fabricated from the as-prepared hierarchical $\mathrm{ZnO}$ nanoflowers versus various operating temperatures from 160 to $440^{\circ} \mathrm{C}$ in pure $\mathrm{N}_{2}$. As shown in Figure 6, the resistance value of the sensor decreases with increasing temperature in the whole temperature range, which is an intrinsic characteristic of an $\mathrm{n}$-type semiconductor gas sensor.

Figure 7 illustrates the gas responses of the fabricated sensors to $30 \mathrm{ppm}$ and $60 \mathrm{ppm}$ of $\mathrm{SO}_{2}$ at various working temperatures. As shown in Figure 7, each response curve of the sensor increases rapidly and achieves the maximum value and then decreases quickly with further increasing temperature. The optimum operating temperature of the fabricated hierarchical $\mathrm{ZnO}$ nanoflowers sensor to $\mathrm{SO}_{2}$ gas was measured at $260^{\circ} \mathrm{C}$, where the sensor exhibits the maximum gas response. And the corresponding maximum response value is 16.72 and 26.14 , respectively. 


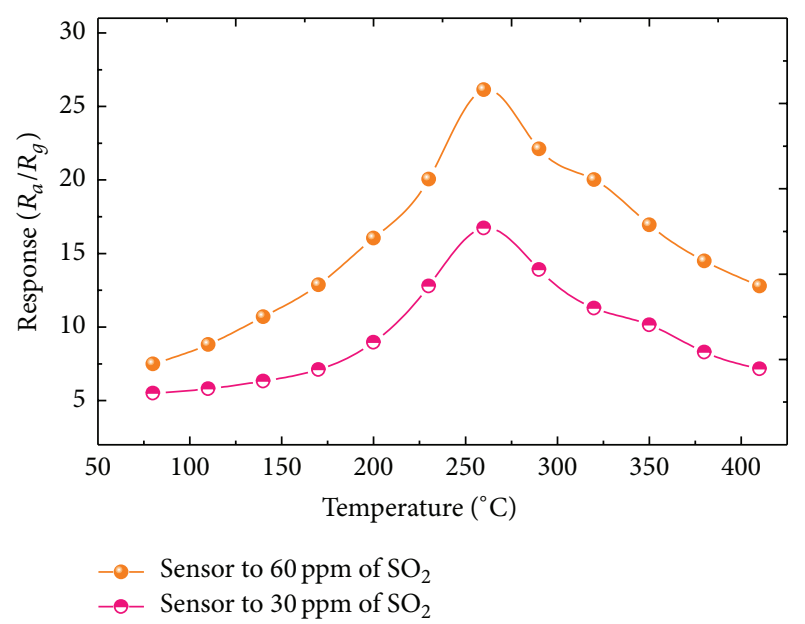

Figure 7: Gas response of the hierarchical $\mathrm{ZnO}$ nanoflowers sensor to 30 and $60 \mathrm{ppm}$ of $\mathrm{SO}_{2}$ at various working temperatures.

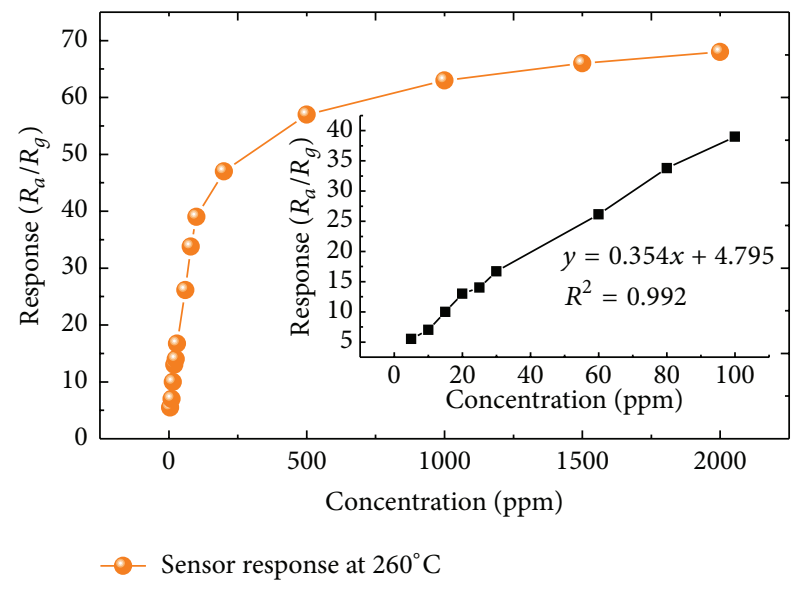

FIGURE 8: Gas response of the hierarchical $\mathrm{ZnO}$ nanoflowers sensor to various concentrations of $\mathrm{SO}_{2}$ at $260^{\circ} \mathrm{C}$.

Figure 8 shows the gas response of the sensor as a function of $\mathrm{SO}_{2}$ gas concentration in the range of $5 \sim 2000 \mathrm{ppm}$, where the sensor worked at its own optimum operating temperature mentioned above. As represented, the sensing responses of the sensor versus $\mathrm{SO}_{2}$ gas increase greatly with increasing gas concentration in the range of 5-100 ppm and achieve saturation when exposed to more than $2000 \mathrm{ppm}$. The saturated gas sensing response value of the sensor to $\mathrm{SO}_{2}$ gas was measured to be about 67.41 .

Figure 9 gives the dynamic response and recovery curve of the sensor versus $80 \mathrm{ppm} \mathrm{SO}_{2}$ to investigate its responserecovery feature. As seen in Figure 9, when certain concentration of $\mathrm{SO}_{2}$ gas was injected into the test chamber, gas response of the sensor increases rapidly and dramatically decreases when the sensor was exposed for recovering, which is an intrinsic characteristic for $\mathrm{n}$-type semiconductor material. Meanwhile, the sensor response returns to its initial value after testing, implying a satisfying stability of the prepared sensor. According to the definition above, the response and

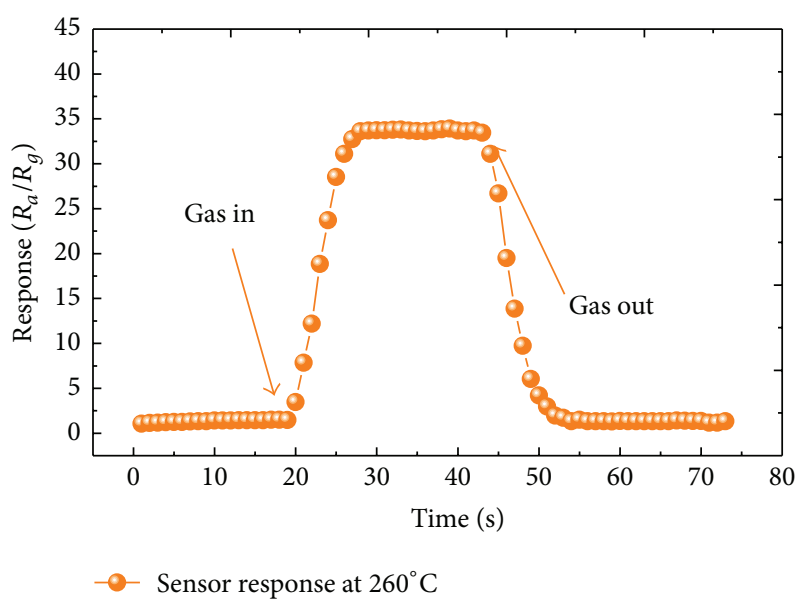

FIgure 9: Dynamic sensing transient of the hierarchical $\mathrm{ZnO}$ nanoflowers sensor to $80 \mathrm{ppm}$ of $\mathrm{SO}_{2}$ at $260^{\circ} \mathrm{C}$.

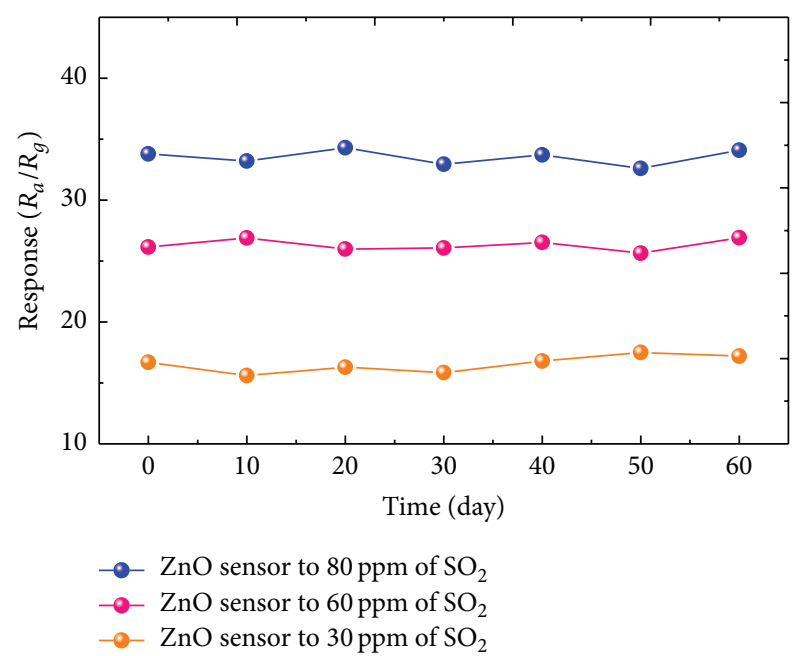

FIGURE 10: Stability of the hierarchical $\mathrm{ZnO}$ nanoflowers sensor to 30,60 , and $80 \mathrm{ppm}$ of $\mathrm{SO}_{2}$ at $260^{\circ} \mathrm{C}$.

recovery times of the sensor are calculated to be about $7 \mathrm{~s}$ and $8 \mathrm{~s}$, respectively.

Finally, the long-term stability of the fabricated hierarchical $\mathrm{ZnO}$ nanoflowers sensor to 30, 60, and $80 \mathrm{ppm}$ of $\mathrm{SO}_{2}$ at $260^{\circ} \mathrm{C}$ was measured and displayed in Figure 10. As seen from the stability plot, the gas response of sensor changes slightly and keeps at a nearly constant value during the long experimental cycles, confirming an excellent longtime stability and repeatability of the sensor for $\mathrm{SO}_{2}$ detection.

\section{Conclusions}

In summary, hierarchical $\mathrm{ZnO}$ nanoflowers were synthesized via hydrothermal method and characterized by XRD, SEM, EDS, and XPS, respectively. Planar chemical gas sensors based on the as-prepared powders were fabricated with screen-printing technique, and their responsive characteristics towards $\mathrm{SO}_{2}$, a most important $\mathrm{SF}_{6}$ decomposition 
byproduct under partial discharge, were systematically investigated with the CGS-1TP intelligent gas sensing analysis system. Lower operating temperature, higher sensing response, good linearity, quick response-recovery characteristic, and good stability were measured with the fabricated planar chemical sensor. All results demonstrate that the sensor fabricated with hierarchical $\mathrm{ZnO}$ nanoflowers is a promising candidate for detecting $\mathrm{SO}_{2}$.

\section{Competing Interests}

The authors declare that there are no competing interests regarding the publication of this paper.

\section{Acknowledgments}

This work has been supported in part by the National Natural Science Foundation of China (no. 51507144), China Postdoctoral Science Foundation funded project (no. 2015M580771), Postdoctoral Science Funded Project of Chongqing (no. Xm2015016), and Fundamental Research Funds for the Central Universities (nos. XDJK2015B005 and SWU114051).

\section{References}

[1] J. Tang, F. Liu, X. X. Zhang, Q. H. Meng, and J. B. Zhou, "Partial discharge recognition through an analysis of $\mathrm{SF}_{6}$ decomposition products part 1: decomposition characteristics of $\mathrm{SF}_{6}$ under four different partial discharges," IEEE Transactions on Dielectrics and Electrical Insulation, vol. 19, no. 1, pp. 29-36, 2012.

[2] J. Tang, F. Liu, Q. H. Meng, X. X. Zhang, and J. G. Tao, "Partial discharge recognition through an analysis of $\mathrm{SF}_{6}$ decomposition products part 2: feature extraction and decision tree-based pattern recognition," IEEE Transactions on Dielectrics and Electrical Insulation, vol. 19, no. 1, pp. 37-44, 2012.

[3] W.-T. Tsai, "The decomposition products of sulfur hexafluoride (SF6): reviews of environmental and health risk analysis," Journal of Fluorine Chemistry, vol. 128, no. 11, pp. 1345-1352, 2007.

[4] C. T. Dervos and P. Vassiliou, "Sulfur hexafluoride $\left(\mathrm{SF}_{6}\right)$ : global environmental effects and toxic byproduct formation," Journal of the Air and Waste Management Association, vol. 50, no. 1, pp. 137-141, 2000.

[5] J. I. Baumbach, P. Pilzecker, and E. Trindade, "Monitoring of circuit breakers using ion mobility spectrometry to detect $\mathrm{SF}_{6}$ decomposition," International Journal for Ion Mobility Spectrometry, vol. 2, no. 1, pp. 35-39, 1999.

[6] R. Kurte, C. Beyer, H. M. Heise, and D. Klockow, "Application of infrared spectroscopy to monitoring gas insulated high-voltage equipment: electrode material-dependent SF6 decomposition," Analytical and Bioanalytical Chemistry, vol. 373, no. 7, pp. 639646, 2002.

[7] D. Velasco-Arias, D. Díaz, P. Santiago-Jacinto, G. RodríguezGattorno, A. Vázquez-Olmos, and S. E. Castillo-Blum, "Direct interaction of colloidal nanostructured $\mathrm{ZnO}$ and $\mathrm{SnO}_{2}$ with $\mathrm{NO}$ and $\mathrm{SO}_{2}$," Journal of Nanoscience and Nanotechnology, vol. 8, no. 12, pp. 6389-6397, 2008.

[8] X. X. Zhang, J. B. Zhang, Y. C. Jia, P. Xiao, and J. Tang, “ $\mathrm{TiO}_{2}$ nanotube array sensor for detecting the $\mathrm{SF}_{6}$ decomposition product $\mathrm{SO}_{2}$," Sensors, vol. 12, no. 3, pp. 3302-3313, 2012.
[9] S. D. Peng, G. L. Wu, and W. Song, "Synthesis, characteristics and enhanced sulfur dioxide sensing properties of $\mathrm{Cu}$-doped $\mathrm{SnO}_{2}$ microspheres," Sensors \& Transducers, vol. 156, no. 9, pp. 310-316, 2013.

[10] J. W. Gong and X. F. Wan, "Electrospun $\mathrm{ZnO}$ nanofibers for selectively detecting $\mathrm{SF}_{6}$ decomposition byproduct $\mathrm{SO}_{2}$," Sensor Letters, vol. 13, no. 10, pp. 857-861, 2015.

[11] X. X. Zhang, J. B. Zhang, J. Tang, and B. Yang, "Gas-sensing simulation of single-walled carbon nanotubes applied to detect gas decomposition products of $\mathrm{SF}_{6}$," Journal of Computational and Theoretical Nanoscience, vol. 9, no. 8, pp. 1096-1100, 2012.

[12] Q. Zhou, W. Chen, S. Peng, and W. Zeng, "Hydrothermal synthesis and acetylene sensing properties of variety low dimensional zinc oxide nanostructures," The Scientific World Journal, vol. 2014, Article ID 489170, 8 pages, 2014.

[13] Q. Qi, T. Zhang, Q. Yu et al., "Properties of humidity sensing $\mathrm{ZnO}$ nanorods-base sensor fabricated by screen-printing," Sensors and Actuators B: Chemical, vol. 133, no. 2, pp. 638-643, 2008.

[14] Z. H. Jing and J. H. Zhan, "Fabrication and gas-sensing properties of porous $\mathrm{ZnO}$ nanoplates," Advanced Materials, vol. 20, no. 23, pp. 4547-4551, 2008.

[15] Q. Zhou, M. Q. Cao, W. D. Li, C. Tang, and S. P. Zhu, "Research on acetylene sensing properties and mechanism of $\mathrm{SnO}_{2}$ based chemical gas sensor decorated with $\mathrm{Sm}_{2} \mathrm{O}_{3}$," Journal of Nanotechnology, vol. 2015, Article ID 714072, 7 pages, 2015.

[16] W. Zeng, T. M. Liu, D. J. Liu, and E. J. Han, "Hydrogen sensing and mechanism of M-doped $\mathrm{SnO}_{2}\left(\mathrm{M}=\mathrm{Cr}^{3+}, \mathrm{Cu}^{2+}\right.$ and $\left.\mathrm{Pd}^{2+}\right)$ nanocomposite," Sensors and Actuators B: Chemical, vol. 160, no. 1, pp. 455-462, 2011.

[17] Q. Qi, T. Zhang, L. Liu, and X. Zheng, "Synthesis and toluene sensing properties of $\mathrm{SnO}_{2}$ nanofibers," Sensors and Actuators B: Chemical, vol. 137, no. 2, pp. 471-475, 2009.

[18] W. Zeng, T. M. Liu, and Z. C. Wang, "Enhanced gas sensing properties by $\mathrm{SnO}_{2}$ nanosphere functionalized $\mathrm{TiO}_{2}$ nanobelts," Journal of Materials Chemistry, vol. 22, no. 8, pp. 3544-3548, 2012.

[19] J. Singh, A. Mukherjee, S. K. Sengupta, J. Im, G. W. Peterson, and J. E. Whitten, "Sulfur dioxide and nitrogen dioxide adsorption on zinc oxide and zirconium hydroxide nanoparticles and the effect on photoluminescence," Applied Surface Science, vol. 258, no. 15, pp. 5778-5785, 2012.

[20] W. Zeng, T. Liu, Z. Wang, S. Tsukimoto, M. Saito, and Y. Ikuhara, "Selective detection of formaldehyde gas using a Cd-Doped $\mathrm{TiO}_{2}-\mathrm{SnO}_{2}$ sensor," Sensors, vol. 9, no. 11, pp. 9029-9038, 2009.

[21] K. Zheng, L. Gu, D. Sun, X. Mo, and G. Chen, "The properties of ethanol gas sensor based on $\mathrm{Ti}$ doped $\mathrm{ZnO}$ nanotetrapods," Materials Science and Engineering B: Solid-State Materials for Advanced Technology, vol. 166, no. 1, pp. 104-107, 2010.

[22] A. Wei, Z. Wang, L.-H. Pan et al., "Room-temperature $\mathrm{NH}_{3}$ gas sensor based on hydrothermally grown $\mathrm{ZnO}$ nanorods," Chinese Physics Letters, vol. 28, no. 8, pp. 702-706, 2011.

[23] Q. Zhou, W. G. Chen, L. N. Xu, and S. D. Peng, "Hydrothermal synthesis of various hierarchical $\mathrm{ZnO}$ nanostructures and their methane sensing properties," Sensors, vol. 13, no. 5, pp. 61716182, 2013.

[24] M. Forouzani, H. R. Mardani, M. Ziari, A. Malekzadeh, and P. Biparva, "Comparative study of oxidation of benzyl alcohol: influence of $\mathrm{Cu}$-doped metal cation on nano $\mathrm{ZnO}$ catalytic activity," Chemical Engineering Journal, vol. 275, no. 9, pp. 220226, 2015. 
[25] M. Israr-Qadir, S. Jamil-Rana, O. Nur, M. Willander, L. A. Larsson, and P. O. Holtz, "Fabrication of $\mathrm{ZnO}$ nanodisks from structural transformation of $\mathrm{ZnO}$ nanorods through natural oxidation and their emission characteristics," Ceramics International, vol. 40, no. 1, pp. 2435-2439, 2014.

[26] J. Guo, J. Zhang, M. Zhu, D. Ju, H. Xu, and B. Cao, "Highperformance gas sensor based on $\mathrm{ZnO}$ nanowires functionalized by Au nanoparticles," Sensors and Actuators B: Chemical, vol. 199, pp. 339-345, 2014.

[27] X. B. Li, S. Y. Ma, F. M. Li et al., "Porous spheres-like ZnO nanostructure as sensitive gas sensors for acetone detection," Materials Letters, vol. 100, no. 6, pp. 119-123, 2013. 

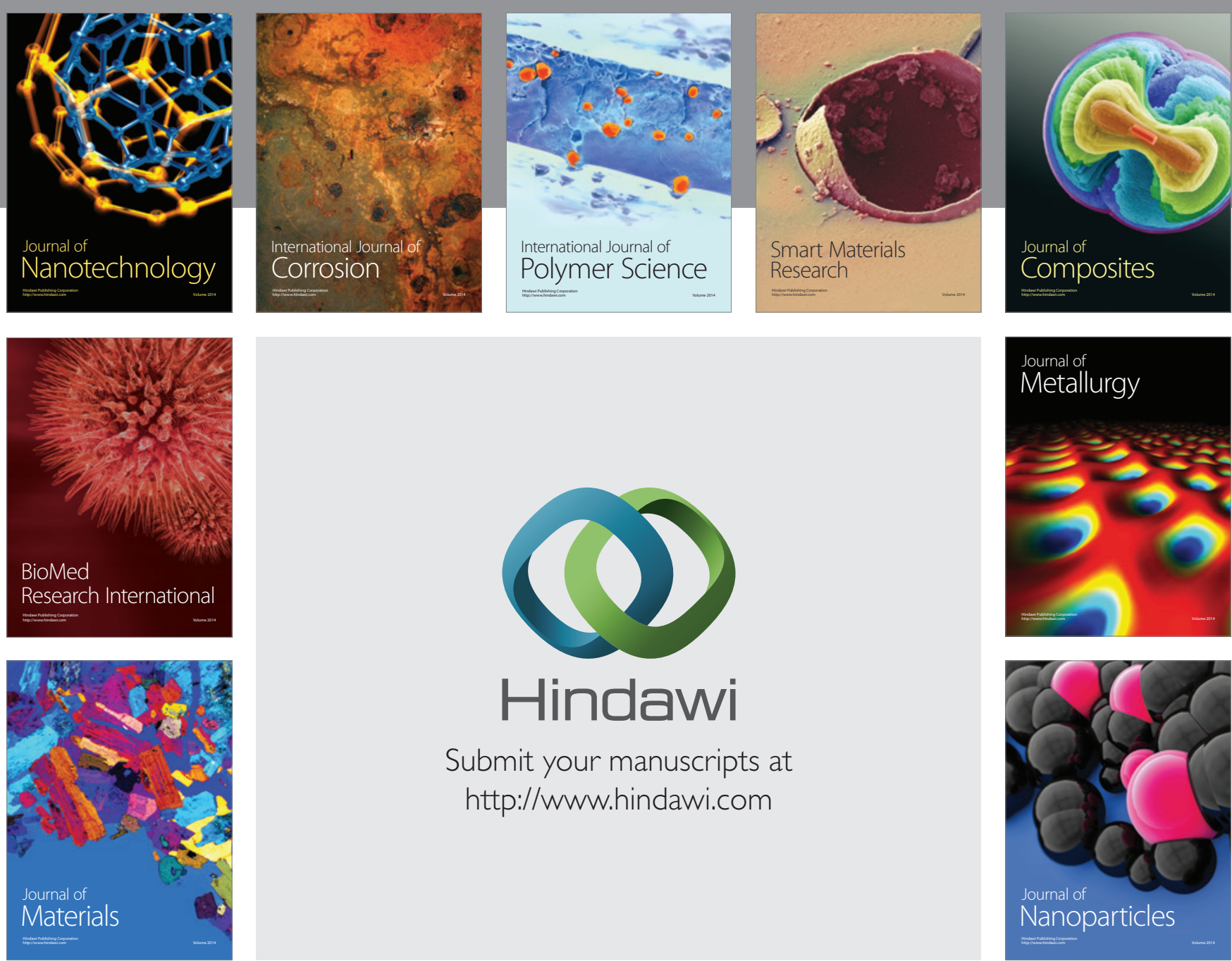

\section{Hindawi}

Submit your manuscripts at

http://www.hindawi.com

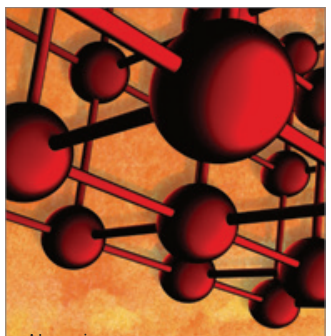

Materials Science and Engineering
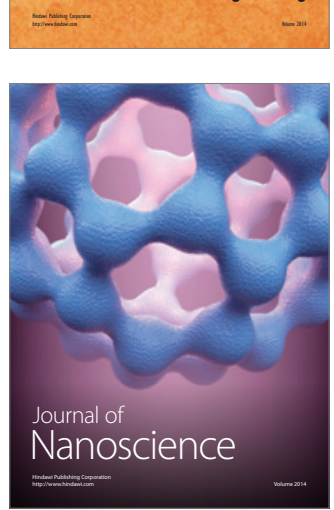
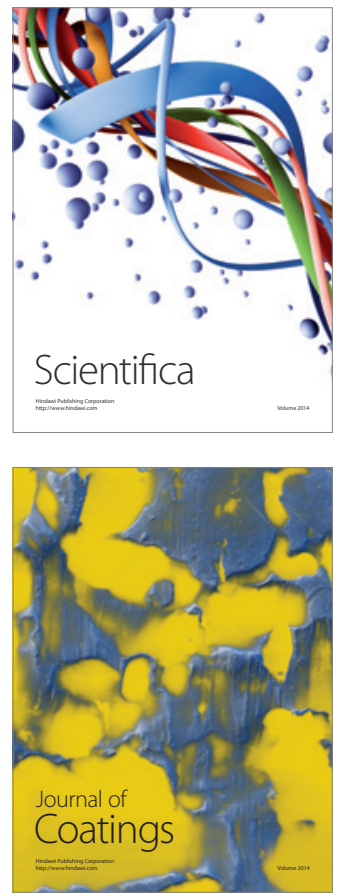
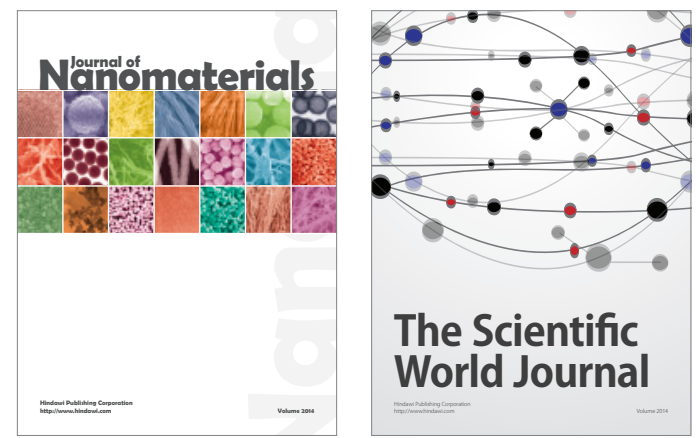

The Scientific World Journal
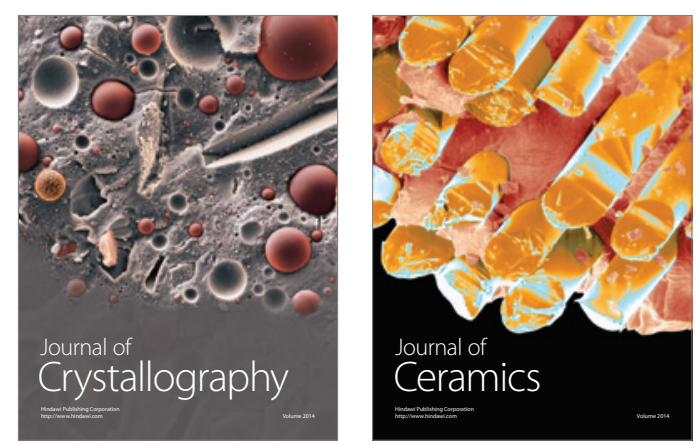
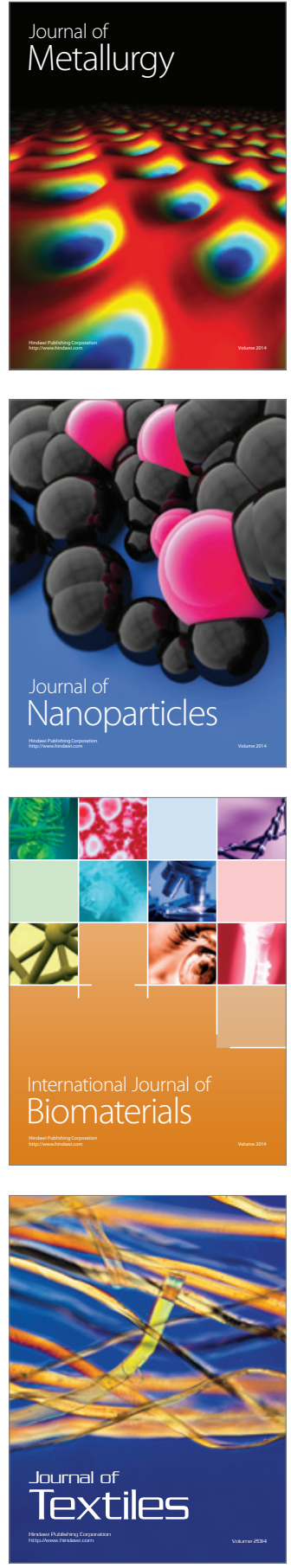